\title{
Lyapunov and Dynamical Spectra for Banach State-spaces
}

\author{
Étienne Voutaz ${ }^{1,2}$
}

Received June 30, 2005; revised July 12, 2006

\begin{abstract}
We study the Lyapunov exponents and exponential splittings for continuous linear skew-product flows acting on infinite dimensional Banach state-spaces.
\end{abstract}

KEY WORDS: Lyapunov exponent; dynamical spectrum; skew-product flow; exponential splitting; evolution semigroup.

MATHEMATICS SUBJECT CLASSIFICATION (2000): Primary: 37H15; 37D20; Secondary: 47D06

\section{INTRODUCTION}

If one thinks to extend the Multiplicative Ergodic Theorem [1,2] to an infinite dimensional space, one could expect that the only change will be the possible existence of infinitely many Lyapunov exponents. However, the spectral theory of bounded linear operators shows that things are not that simple.

Let $T$ be a bounded linear operator of the Banach space $E$. The essential spectral radius $\rho_{\mathrm{ess}}(T)$ is the smallest number $r \geqslant 0$ such that any $\lambda$ in the spectrum of $T$ with modulus larger than $r$ is an isolated eigenvalue of finite (algebraic) multiplicity. We note Nussbaum's formula [3]:

$$
\rho_{\text {ess }}(T)=\lim _{n \rightarrow \infty}\left(\inf \left\{\left\|T^{n}-K\right\| \mid K \in \mathcal{L}(E) \text { is compact }\right\}\right)^{1 / n}
$$

\footnotetext{
${ }^{1}$ Mathematik Departement, Eidgenössische Technische Hochschule, ETH-Zürich, CH-8092 Zürich, Switzerland.

${ }^{2}$ Current address: HEVs, Rte de la Plaine 2, CH-3960 Sierre, Switzerland. E-mail: etienne.voutaz@hevs.ch
} 
for the essential spectral radius, giving the smallest disk containing the Browder essential spectrum. There is thus no hope to find Lyapunov exponents smaller than the essential spectral radius. The most satisfactory situation is when the cocyle is measurable and consists of compact operators, and a Multiplicative Ergodic Theorem was proved by Mañé in this context (see [4]). The theorem was then improved by Thieullen [5] for "quasi-compact" measurable cocycles; Thieullen introduced a measurable index of compactness similar to the essential spectral radius.

On the other hand, if we consider continuous cocycles (see [6]), we can obtain an exponential splitting corresponding to the different growthrates of vectors in $E$. It was then proved by Sacker and Sell [7] that, under some recurrence properties in the base space, for numbers $\theta$ larger than a similar uniform index of compactness, $\theta$ is not in the dynamical spectrum if, and only if, there are no nonzero vectors with positive and negative shifted orbits uniformly bounded (see Theorems 1 and 2). The corresponding expanding subspaces are moreover finite dimensional.

The aim of this work is to study the relations between the (measurable) Lyapunov spectrum and the dynamical spectrum (corresponding to the usual spectrum for bounded linear operators). We prove in particular that

- The uniform index of compactness is equal to the supremum of the measurable ones. The supremum is moreover attained for some invariant, ergodic Borel probability measure (Theorem 5).

- The boundary points of the compact intervals of the dynamical spectrum correspond to Lyapunov exponents for invariant, ergodic Borel probability measures (Theorem 5).

These are generalizations of results appearing in $[8,9]$.

\section{SKEW-PRODUCT FLOWS}

\subsection{Cocycles and Skew-Product Flows}

Let $\phi: X \rightarrow X$ be a homeomorphism of the compact metric space $X,(E,\|\cdot\|)$ a (real) Banach space and $x \mapsto \Phi(x)$ a mapping from $X$ into $\mathcal{L}(E)$, continuous in the norm topology of $\mathcal{L}(E)$. We construct from these objects a mapping $(n, x) \mapsto \Phi(n, x)$, defined for $n$ a nonnegative integer and $x \in X$ by

$$
\Phi(0, x)=\mathrm{id}, \quad \Phi(n, x)=\Phi(n-1, \phi x) \Phi(x) .
$$

Then $x \mapsto \Phi(n, x)$ is continuous for each $n$, and the cocycle identity

$$
\Phi(n+m, x)=\Phi\left(n, \phi^{m} x\right) \Phi(m, x),
$$


holds. We will indifferently refer to $x \mapsto \Phi(x)$ and $x \mapsto \Phi(n, x)$ as a cocycle. (It would be more precise to say continuous semicocycle, but we will use the shorter terminology.)

We will allow ourselves to use the word "vector" to refer either to elements of $E$ or to elements $(x, v)$ of $X \times E$. In the latter case, words like "norm", "normalized", "bounded", etc. refer only to the $v$ component.

The definition of $\Phi(n, x)$ is motivated by the following construction: Given $\phi: X \rightarrow X$ and $\Phi: X \rightarrow \mathcal{L}(E)$, we define the semidirect product mapping $\pi$ of $X \times E$ to itself by $\pi(x, v)=(\phi x, \Phi(x) v)$. It is then easy to see that $\pi^{n}(x, v)=\left(\phi^{n} x, \Phi(n, x) v\right)$. We will refer to $\pi$ as a linear skew-product semiflow ( skew-product flow for short), having in mind the applications to differential equations.

If - and only if - each $\Phi(x)$ is invertible, $\pi$ is also invertible; the definition of $\Phi(n, x)$ can then be extended to negative $n$ in such a way that the cocycle identity and the relation between $\pi^{n}$ and $\Phi(n, x)$ continue to hold. In the applications we have in mind, $E$ will be infinite dimensional and the $\Phi(x)$, although bounded, will almost never have bounded inverses. For this reason, we need to write all formulas in terms of the $\Phi(n, x)$ for nonnegative $n$, although this will sometimes make them less transparent than one might wish.

With this in mind we define a negative continuation of a vector $\left(x_{0}, v_{0}\right)$ - or sometimes of the vector $v_{0}$ at $x_{0}$ - to be the sequence of vectors $\left(x_{-1}, v_{-1}\right),\left(x_{-2}, v_{-2}\right), \ldots$ such that $\pi\left(x_{-n}, v_{-n}\right)=\left(x_{-n+1}, v_{-n+1}\right)$ for $n=1,2, \ldots$ In other words

- $\phi x_{-n}=x_{-n+1}$, i.e., $x_{-n}=\phi^{-n} x_{0}$,

- $\Phi\left(x_{-n}\right) v_{-n}=v_{-n+1}$.

A given vector may or may not admit a negative continuation; if it does, its negative continuation may or may not be unique. If $\Phi(x)$ is invertible for all $x$, then every vector $\left(x_{0}, v_{0}\right)$ has a unique negative continuation, namely $\pi^{-n}\left(x_{0}, v_{0}\right)$.

Remark 1. If $\pi=(\phi, \Phi)$ is a continuous-time skew-product flow that is continuous in norm, i.e., each map $(t, x) \mapsto \Phi(t, x) \in \mathcal{L}(E)$ is continuous, then each operator $\Phi(t, x)$ is invertible and the cocycle identity holds for negative times also. In this setting, we hence usually work with strongly continuous skew-product flows, i.e., those with each map $(t, x, v) \mapsto \Phi(t, x) v \in E$ continuous (see [10, Remark 6.2]).

We introduce now a useful device: If $\Phi(n, x)$ is a cocycle, then so is $\Phi_{\theta}(n, x)$ defined by $\Phi_{\theta}(n, x):=e^{-n \theta} \Phi(n, x)$ for every real number $\theta$. We will refer to $\Phi_{\theta}$ as the cocycle $\Phi$ shifted by $\theta$ and also consider the shifted semidirect product mapping $\pi_{\theta}$. However, when we will consider properties 
of a negative continuation we will always have in mind "of the unshifted cocycle".

\section{SUBBUNDLES AND HYPERBOLIC SPLITTINGS}

We want now to give a precise definition of hyperbolicity. To do this, some preliminary concepts are needed: Let $X$ and $E$ be as before; let $\mathcal{E}$ be a subset of $X \times E$ and $Y$ a compact subset of $X$. We define the fiber $\mathcal{E}(x)$ of $\mathcal{E}$ over $x$ by

$$
\mathcal{E}(x):=\{v \in E \mid(x, v) \in \mathcal{E}\} .
$$

A subset $\mathcal{E}$ of $X \times E$ will be called a subbundle over $Y$ if, for each $y \in Y$,

(1) the fiber $\mathcal{E}(y)$ is closed in $E$,

(2) $\mathcal{E}(y)$ has a closed complement $\mathcal{F}(y)$ in $E$, i.e., $E=\mathcal{E}(y) \oplus \mathcal{F}(y)$. We will also write the direct sum as a Whitney sum: $Y \times E=$ $\mathcal{E} \oplus \mathcal{F}$.

We will moreover say that the splitting $Y \times E=\mathcal{E} \oplus \mathcal{F}$ is continuous if, denoting by $P(y)$ the projection onto $\mathcal{E}(y)$ along $\mathcal{F}(y)$, the mapping $y \mapsto P(y)$ is norm continuous from $Y$ into $\mathcal{L}(E)$. In the applications we have in mind we are mostly interested in one of the subbundles, say $\mathcal{E}$; we will abuse language in this case and say that the subbundle $\mathcal{E}$ is continuous over $Y$, without referring to $\mathcal{F}$.

Let $\pi=(\phi, \Phi)$ be a skew-product flow. A subbundle $\mathcal{E}$ over $Y$ is called $\pi$-invariant if

(1) $Y$ is $\phi$-invariant, i.e., $\phi(Y)=Y$,

(2) $\Phi(y) \mathcal{E}(y) \subset \mathcal{E}(\phi y)$ for all $y \in Y$,

(3) $\Phi(y) \mathcal{F}(y) \subset \mathcal{F}(\phi y)$ for all $y \in Y$ (F denotes the complementary subbundle of $\mathcal{E}$ in $Y \times E$ ).

Let:

- The forward $\theta$-bounded set $\mathcal{B}_{\theta}^{+}(\mathcal{E})$ be the set of all vectors $(x, v)$ in $\mathcal{E}$ such that $\Phi_{\theta}(n, x) v$ is uniformly bounded for $n=0,1,2, \ldots$; we refer to such an $(x, v)$ as a $\theta$-contracting vector. (It might be better to say "forward $\theta$-bounded vector" instead of " $\theta$-contracting vector". "Contracting" is, however, shorter, and we will see later that, in the case of greatest interest to us, the terminology is justified.)

- The backward $\theta$-bounded set $\mathcal{B}_{\theta}^{-}(\mathcal{E})$ be the set of all vectors $(x, v)$ in $\mathcal{E}$ admitting a negative continuation $\left(x_{-n}, v_{-n}\right)$ in $\mathcal{E}$ with $e^{n \theta} v_{-n}$ uniformly bounded for $n=1,2, \ldots$; such an $(x, v)$ is a 
$\theta$-expanding vector. (As is traditional in this area, "expanding" is to be understood as meaning "contracting backwards".)

- The $\theta$-bounded set $\mathcal{B}_{\theta}(\mathcal{E})$ be $\mathcal{B}_{\theta}^{+}(\mathcal{E}) \cap \mathcal{B}_{\theta}^{-}(\mathcal{E})$; an $(x, v)$ in $\mathcal{B}_{\theta}(\mathcal{E})$ is a $\theta$-bounded vector.

- $\mathcal{O}(\mathcal{E})$ be the set of trivial vectors, i.e., the set of vectors $(x, v)$ in $\mathcal{E}$ such that $v=0$.

We will often write, e.g., $\mathcal{B}_{\theta}$ for $\mathcal{B}_{\theta}(X \times E), \mathcal{B}$ instead of $\mathcal{B}_{0}$ or $\mathcal{B}(\pi)$ to clarify what we have in mind.

We say that the skew-product flow $\pi$ admits a hyperbolic splitting (or an exponential dichotomy) over $Y$ if there exist continuous, $\pi$-invariant subbundles $\mathcal{E}^{u}$ and $\mathcal{E}^{s}$ over $Y$ such that

(1) $Y \times E=\mathcal{E}^{u} \oplus \mathcal{E}^{s}$;

(2) $\operatorname{dim}\left(\mathcal{E}^{u}(y)\right)<\infty$ for all $y \in Y$;

(3) the restriction of $\Phi$ to $\mathcal{E}^{u}$ is invertible (and hence $\Phi(y) \mathcal{E}^{u}(y)=$ $\left.\mathcal{E}^{u}(\phi y)\right)$;

(4) there exist positive constants $c, \beta$ such that

$$
\begin{gathered}
\|\Phi(n, y) v\| \leqslant c e^{-n \beta}\|v\| \quad \text { for all } n \geqslant 0,(y, v) \in \mathcal{E}^{s}, \\
\|\Phi(-n, y) v\| \leqslant c e^{-n \beta}\|v\| \quad \text { for all } n \geqslant 0,(y, v) \in \mathcal{E}^{u} .
\end{gathered}
$$

The subbundles $\mathcal{E}^{s}$ and $\mathcal{E}^{u}$ are called the stable and unstable subbundles respectively.

Remark 2. We stress that the finite dimensionality of the unstable subbundle is a strong condition and is part of our definition of a hyperbolic splitting. In this case, we can replace condition (1) by the following equivalent one:

$$
\begin{aligned}
& \|\Phi(n, y) v\| \leqslant c e^{-n \beta}\|v\| \text { for all } n \geqslant 0,(y, v) \in \mathcal{E}^{s}, \\
& \|\Phi(n, y) v\| \geqslant c^{-1} e^{n \beta}\|v\| \text { for all } n \geqslant 0,(y, v) \in \mathcal{E}^{u} .
\end{aligned}
$$

We cannot obtain a Multiplicative Ergodic Theorem without such properties (see Theorem 3, more precisely the definition of the measurable index of compactness). Moreover, the Sacker-Sell characterization of hyperbolicity (Theorem 1) only works in this context.

\section{EXPONENTIAL SPLITTINGS}

\subsection{Index of Compactness}

Let $(M, d)$ be a metric space. We denote by $B_{M}(v, r)$ the open ball of radius $r$ in $M$ and centered at $v \in M$. Following Kuratowski [11] we define 
for every bounded subset $Y$ of $M$ a number $\alpha(Y)$ as the infimum of all reals $r>0$ such that $Y$ can be covered by a finite number of open balls with centers in $Y$ and radius $r$. We note the following properties of $\alpha$ :

- $\alpha(Y)=0 \Leftrightarrow \bar{Y}$ is compact.

- If $E$ is a real normed space then $\operatorname{dim}(E)=\infty$ if, and only if, $\alpha\left(B_{E}(0,1)\right)=1$.

Let $(E,\|\|$.$) be a real normed space and A \in \mathcal{L}(E)$. We define the index of compactness $\varrho(A)$ of $A$ by $\varrho(A):=\alpha\left(A\left(B_{E}(0,1)\right)\right)$. We note that $\varrho(A) \leqslant\|A\|$ and that $A$ is a compact operator on a Banach space $E$ if, and only if, $\varrho(A)=0$ (see [3] for properties of $\varrho$ ).

We define the (logarithmic) index of (uniform asymptotic) compactness $\kappa(\pi) \in[-\infty,+\infty)$ of the skew-product flow $\pi=(\phi, \Phi)$ by

$$
\kappa(\pi):=\liminf _{n \rightarrow+\infty} \frac{1}{n} \log \sup _{x \in X} \varrho(\Phi(n, x)),
$$

and also

$$
\kappa_{\mathrm{ess}}(\pi):=\liminf _{n \rightarrow+\infty} \frac{1}{n} \log \sup _{x \in X} \inf \{\|\Phi(n, x)-K\| \mid K \in \mathcal{L}(E) \text { compact }\} .
$$

The number $\kappa_{\mathrm{ess}}(\pi)$ will be called the essential (logarithmic) index of (uniform asymptotic) compactness. The Subadditive Lemma [12, Theorem 4.9] yields, e.g.,

$$
\kappa(\pi)=\lim _{n \rightarrow+\infty} \frac{1}{n} \log \sup _{x \in X} \varrho(\Phi(n, x))=\inf _{n \geqslant 1} \frac{1}{n} \log \sup _{x \in X} \varrho(\Phi(n, x)) .
$$

We also introduce a number $\bar{\mu}(\pi)$ defined as the infimum of all reals $\zeta$ such that $\left\|\Phi_{\zeta}(n, x)\right\|$ is bounded over $n=0,1, \ldots$ and $x \in X$.

Proposition 1. Let $E$ be a Banach space, A a bounded, linear operator in $E$ and $\pi$ a skew-product flow over the compact metric space $X$. Then

(1) $\varrho(A) \leqslant \inf \{\|A-K\| \mid K \in \mathcal{L}(E)$ is a compact operator $\}$;

(2) $\kappa(\pi) \leqslant \kappa_{\mathrm{ess}}(\pi) \leqslant \bar{\mu}(\pi)$.

Proof. The proof follows from standard properties of the Kuratowski functional $\alpha$.

We do not know examples with $\kappa(\pi)<\kappa_{\mathrm{ess}}(\pi)$. However, we can prove that $\kappa(\pi)=\kappa_{\text {ess }}(\pi)$ under some assumptions on the Banach space $E$ (see Lemma 1). 


\subsection{The Sacker-Sell Theorem}

The result below was proved by Sacker and Sell [7, Theorem B, p. 28] for strongly continuous, continuous-time cocycles over a flow. We recall that a homemorphism $\phi$ of the compact metric space $X$ is one-sided topologically transitive if there exists a dense orbit $\left\{\phi^{n}(x) \mid n \geqslant 0\right\}$ in $X$.

Theorem 1. (Sacker-Sell). Suppose that the skew-product flow $\pi=(\phi, \Phi)$ satisfies the following condition over the compact metric space X:

(H1) $\phi: X \rightarrow X$ is one-sided topologically transitive.

Then the system $\pi=(\phi, \Phi)$ admits a hyperbolic splitting over $X$ if, and only if, $\mathcal{B}(\pi)=\mathcal{O}$ and $\lim _{n \rightarrow \infty} \sup _{x \in X} \varrho(\Phi(n, x))=0$.

It may at first seem surprising that it suffices to assume

$$
\lim _{n \rightarrow \infty} \sup _{x \in X} \varrho(\Phi(n, x))=0,
$$

rather than the apparently stronger condition $\kappa(\pi)<0$, i.e., the exponential falloff with $n$ of $\sup _{x} \varrho(\Phi(n, x))$. In fact, however, the two hypotheses are equivalent: The mapping $n \mapsto \log \sup _{x} \varrho(\Phi(n, x))$ is subadditive, so

$$
\lim _{n \rightarrow \infty} \frac{1}{n} \log \sup _{x} \varrho(\Phi(n, x))=\inf _{n} \frac{1}{n} \log \sup _{x} \varrho(\Phi(n, x)) .
$$

Thus, if there exists one $n_{0}$ such that $\sup _{x} \varrho\left(\Phi\left(n_{0}, x\right)\right)<1$, then $\sup _{x} \varrho(\Phi(n$, $x$ )) falls off exponentially with $n$.

Sacker and Sell first proved the theorem for a finite-dimensional Banach space [13-15]. In order to treat the infinite-dimensional case, some compactness conditions (smoothing properties) are needed to prove the invertibility of $\Phi$ acting on the (finite-dimensional) unstable subbundle. Some assumption like (H1) is necessary, even in the finite-dimensional case: A diffeomorphism $f$ of a compact manifold $M$ is often called quasi-Anosov if the bounded set $\mathcal{B}(d f)(x)$ of the differential $d f$ is trivial for all $x \in M$. In the 1970s the following question arose: is a quasi-Anosov diffeomorphism Anosov? This question has a positive answer in dimension two as shown by Mañé [16], but the answer is definitely no in general: Franks and Robinson [1] have exhibited a counterexample in dimension three. However, it became clear that a quasi-Anosov diffeomorphism satisfying some recurrence conditions is Anosov [13, 16, 18].

Remark that assumption (H1) is replaced by the following ones in the work of Sacker and Sell [7, Theorems D and F]:

(H2) The compact metric space $X$ is connected and the union of the minimal sets of $\phi$ is dense in $X$.

(H3) $X$ is connected and $\phi$ is chain recurrent on $X$. 
In particular, (H2) holds true if the periodic points of $\phi$ are dense in $X$. From the probabilistic point of view, we can replace (H1) by the following stronger condition [12, Theorem 5.16]:

(H4) There exists a $\phi$-invariant, ergodic Borel probability measure on $X$ giving nonzero measure to every nonempty open subset of $X$.

If recurrence properties as $(\mathrm{H} 1),(\mathrm{H} 2),(\mathrm{H} 3)$ or $(\mathrm{H} 4)$ do not hold, we can nevertheless decompose the space $X$ into finitely many compact, invariant Morse sets over each of which the skew-product flow $\pi$ admits a hyperbolic splitting [7, Theorem E].

It is possible to generalize Theorem 1 in the direction of Theorem 2 below, using the shifted systems $\pi_{\theta}$. This approach was adopted by Chow and Leiva [19] for continuous-time cocycles.

\subsection{Dynamical Spectrum and Exponential Splitting}

Let $\phi$ be a homeomorphism of the compact metric space $X$ and $\pi=(\phi, \Phi)$ a continuous skew-product flow over $X$. We define a subset $\operatorname{Res}(\pi)$ of the reals, the resolvent set of $\pi$, by

$$
\operatorname{Res}(\pi):=\left\{\theta \in \mathbb{R} \mid \pi_{\theta} \text { admits a hyperbolic splitting over } X\right\} \text {. }
$$

The dynamical spectrum $\Sigma(\pi)$ (or Sacker-Sell spectrum) is defined as the complement of the resolvent set in the reals, i.e., $\Sigma(\pi):=\mathbb{R} \backslash \operatorname{Res}(\pi)$. An interval of the kind $(-\infty, a]$ will be conventionally empty if $a=-\infty$. We stress that "hyperbolic splitting" means, in particular, that the unstable bundle is finite-dimensional. It is easy to see that the resolvent set $\operatorname{Res}(\pi)$ is an open subset of the reals, and hence, the dynamical spectrum $\Sigma(\pi)$ is closed.

Theorem 2. (Bounded Sets and Exponential Splittings). Let (E, $\|\|$. be an infinite-dimensional (real) Banach space, $\pi$ a continuous skew-product flow over the compact metric space $X$ and suppose $\phi: X \rightarrow X$ is onesided topologically transitive. Assume that $\Sigma(\pi) \neq \emptyset, \quad \Sigma(\pi) \neq(-\infty, \bar{\mu}(\pi)]$. Then there exist numbers $\bar{\mu}(\pi)=: \mu_{1} \geqslant \lambda_{1}>\mu_{2} \geqslant \lambda_{2}>\cdots>\mu_{j} \geqslant \lambda_{j}>\cdots>$ $\kappa_{+} \geqslant \kappa(\pi)\left(\mu_{j} \searrow \kappa_{+}\right.$if we have (countably) infinitely many such numbers) and families of nontrivial, continuous, $\pi$-invariant subbundles $\left\{\mathcal{H}_{j}\right\},\left\{\widehat{\mathcal{H}}_{k}\right\}$ of $X \times E$ such that

(1) $\Phi(x) \mathcal{H}_{j}(x)=\mathcal{H}_{j}(\phi x) \quad$ for all $x$ and $j$; $\Phi(x) \widehat{\mathcal{H}}_{k}(x) \subset \widehat{\mathcal{H}}_{k}(\phi x)$ for all $x$ and $k$. 
(2) $1 \leqslant \operatorname{dim}\left(\mathcal{H}_{j}(x)\right)=: d_{j}<\infty$ for all $x$ and $j$. In particular, the dimension does not depend on $x$.

(3) $\widehat{\mathcal{H}}_{1}:=X \times E, \quad \widehat{\mathcal{H}}_{j}=\mathcal{H}_{j} \oplus \widehat{\mathcal{H}}_{j+1} \quad$ for all $j$.

(4) $\Sigma(\pi)=\left(-\infty, \kappa_{+}\right] \cup \bigcup_{j}\left[\lambda_{j}, \mu_{j}\right]$.

(5) $\quad \Sigma\left(\pi \mid \mathcal{H}_{j}\right)=\left[\lambda_{j}, \mu_{j}\right], \quad \Sigma\left(\pi \mid \widehat{\mathcal{H}}_{k}\right)=\Sigma(\pi) \cap\left(-\infty, \mu_{k}\right] \quad$ for all $j, k$ and, if there are $p<\infty$ subbundles $\mathcal{H}_{j}$, then $\Sigma\left(\pi \mid \widehat{\mathcal{H}}_{p+1}\right)=$ $\left(-\infty, \kappa_{+}\right]$.

(6) The restriction of $\pi$ to $\mathcal{H}_{j}$ is invertible and there is a constant $c>0$ such that for all $x$ and $j$,

$$
\begin{aligned}
\|\Phi(n, x) v\| & \leqslant c e^{n \mu_{j}}\|v\| \quad \text { for all } v \in \mathcal{H}_{j}(x), n \geqslant 0, \\
\|\Phi(-n, x) v\| & \leqslant c e^{-n \lambda_{j}}\|v\| \quad \text { for all } v \in \mathcal{H}_{j}(x), n \geqslant 0, \\
\left\|\Phi(n, x) \mid \widehat{\mathcal{H}}_{k}(x)\right\| & \leqslant c e^{n \mu_{k}} \quad \text { for all } n \geqslant 0, \text { all } k .
\end{aligned}
$$

If there are $p<\infty$ subbundles $\mathcal{H}_{j}$, then

$$
\left\|\Phi(n, x) \mid \widehat{\mathcal{H}}_{p+1}(x)\right\| \leqslant c e^{n \kappa_{+}} \text {for all } x \text {, all } n \geqslant 0 .
$$

(7) $\theta \in \operatorname{Res}(\pi) \Leftrightarrow \theta>\kappa(\pi)$ and $\mathcal{B}_{\theta}(\pi)=\mathcal{O}$.

We also note that

$$
\bar{\mu}(\pi)=\lim _{n \rightarrow \infty} \frac{1}{n} \log \sup _{x \in X}\|\Phi(n, x)\| .
$$

Proof. See [10, Section 6.3, 19]. A direct proof of the theorem-not assuming Theorem 1 - can be found in [20].

Remark 3. We will prove later in Section 5.2, using Lyapunov exponents, that $\kappa_{+}=\kappa(\pi)$ if we have infinitely many compact intervals $\left[\lambda_{j}, \mu_{j}\right]$ in the dynamical spectrum $\Sigma(\pi)$ (see Corollary 2).

We will call the family $\left\{\mathcal{H}_{j}\right\}$ of Theorem 2 the exponential splitting associated to the skew-product flow $\pi$ and denote it by $\mathcal{S}(\pi):=\left\{\mathcal{H}_{j}=\right.$ $\left.\mathcal{H}_{\lambda_{j}, \mu_{j}}(\pi)\right\}$ to specify the characterizations of the family. The number $\kappa_{+}(\pi):=\kappa_{+}$will be called the dynamical index of compactness of the skewproduct flow $\pi$.

We have clearly $\kappa(\pi) \leqslant \kappa_{\text {ess }}(\pi) \leqslant \kappa_{+}(\pi)$ (see Proposition 1 ). We prove below that, under some assumptions on the Banach space $E$, we always have $\kappa(\pi)=\kappa_{\text {ess }}(\pi)$. We do not know, however, if $\kappa_{\text {ess }}(\pi)<\kappa_{+}(\pi)$ is possible.

Lemma 1. (Compact approximations of the identity). Let $E$ be a Banach space such that there are a sequence of linear operators $\Pi_{j}$ on $E$ and a positive constant $B$ such that 
(1) $\left\|\Pi_{j}\right\| \leqslant B \quad$ for all $j$,

(2) $I-\Pi_{j}$ is compact for all $j$,

(3) $D:=\left\{v \in E \mid \Pi_{j} v \rightarrow 0\right\} \quad$ is dense in $E$.

Let moreover $A$ be a bounded, linear operator on E. Then

$$
\inf _{K}\|A-K\| \leqslant B \varrho(A),
$$

where the infimum is taken over all compact operators $K \in \mathcal{L}(E)$. In particular, $\kappa(\pi)=\kappa_{\mathrm{ess}}(\pi)$ for every skew-product flow $\pi$ on $E$.

Proof. To see this, take a positive $\epsilon$; by definition of $\varrho(\cdot)$, there are vectors $v_{1}, v_{2}, \ldots, v_{n}$ in $E$ such that

$$
A\left(B_{E}(0,1)\right) \subset \bigcup_{i=1}^{n} B_{E}\left(v_{i}, \varrho(A)+\epsilon\right) .
$$

We can now choose vectors $w_{1}, w_{2}, \ldots, w_{n}$ in $D$ such that

$$
\left\|v_{i}-w_{i}\right\|<\frac{\epsilon}{2 B} \quad \text { for } i=1, \ldots, n .
$$

Therefore, we can find some integer $j_{0}=j_{0}(\epsilon)$ such that for all $j \geqslant j_{0}$ and all $i$ :

$$
\left\|\Pi_{j} v_{i}\right\| \leqslant\left\|\Pi_{j} w_{i}\right\|+\left\|\Pi_{j}\left(v_{i}-w_{i}\right)\right\|<\frac{\epsilon}{2}+B \cdot \frac{\epsilon}{2 B} \leqslant \epsilon .
$$

We now claim that $\left\|\Pi_{j} A\right\| \leqslant B(\varrho(A)+\epsilon)+\epsilon$ for all $j \geqslant j_{0}$. Indeed, if $v \in$ $B_{E}(0,1)$ we can assume that $A v \in B_{E}\left(v_{i}, \varrho(A)+\epsilon\right)$ and hence

$$
\left\|\Pi_{j} A v\right\| \leqslant\left\|\Pi_{j}\left(A v-v_{i}\right)\right\|+\left\|\Pi_{j} v_{i}\right\|<B(\varrho(A)+\epsilon)+\epsilon \text { for all } j \geqslant j_{0} .
$$

However, $\left(I-\Pi_{j}\right) A$ is a compact operator for every $j$ and thus

$$
\inf _{K}\|A-K\| \leqslant\left\|A-\left(I-\Pi_{j}\right) A\right\|=\left\|\Pi_{j} A\right\| \leqslant B(\varrho(A)+\epsilon)+\epsilon
$$

for all $j \geqslant j_{0}$. The claim follows since $\epsilon$ is arbitrary.

Such compact approximations of the identity are discussed by Holschneider [21] and Baladi and Holschneider [22], where it is proved, e.g., that

$$
\begin{aligned}
\kappa(\pi)=\kappa_{\mathrm{ess}}(\pi) & =\lim _{n \rightarrow \infty} \frac{1}{n} \log \sup _{x} \limsup _{j}\left\|\Pi_{j} \Phi(n, x)\right\| \\
& =\lim _{n \rightarrow \infty} \frac{1}{n} \log \sup _{x} \liminf _{j}\left\|\Pi_{j} \Phi(n, x)\right\| .
\end{aligned}
$$

A compact approximation of the identity as in Lemma 1 exists in the following spaces (see [22, Lemma 4]): 
(1) The spaces $H^{s}\left(\mathbb{T}^{n}\right), s>0$, of Sobolev mappings defined on the $n$-dimensional torus $\mathbb{T}^{n}$.

(2) The closure in the space $\mathcal{C}^{r}\left(\mathbb{T}^{n}\right)$ of $\mathcal{C}^{\infty}\left(\mathbb{T}^{n}\right), r \geqslant 0$.

The following corollary can be found in [23], where completely continuous skew-product flows are considered, and in [19].

Corollary 1. (Dynamical spectrum). The dynamical spectrum $\Sigma(\pi)$ of the system $\pi$ is exactly one of the following sets:

(1) $\Sigma(\pi)=\emptyset$;

(2) $\Sigma(\pi)=(-\infty, \bar{\mu}(\pi)]$;

(3) $\Sigma(\pi)=\left(-\infty, \kappa_{+}(\pi)\right] \cup \bigcup_{j}\left[\lambda_{j}, \mu_{j}\right]$, where $\bar{\mu}(\pi)=\mu_{1} \geqslant \lambda_{1}>\mu_{2} \geqslant$ $\lambda_{2}>\cdots>\mu_{j} \geqslant \lambda_{j}>\cdots>\kappa_{+}(\pi) \geqslant \kappa_{\mathrm{ess}}(\pi) \geqslant \kappa(\pi)$, and $\mu_{j} \searrow \kappa(\pi)=$ $\kappa_{+}(\pi)$ if we have infinitely many such numbers.

Remark that the spectrum of a compact operator on a Banach space can be equal to $\{0\}$; so, $\Sigma(\pi)=\emptyset$ is possible if the base space $X$ is trivial.

Remark 4. Our definition of a hyperbolic splitting is relatively restrictive and hence the interval $(-\infty, \kappa(\pi)]$ is always contained in the dynamical spectrum $\Sigma(\pi)$. In particular, if we have a "hyperbolic splitting" with infinite dimensional expanding subspaces, we do not consider this as "hyperbolic". In order to generalize this concept, it is however essential that the restriction of $\Phi(n, x)$ to $\operatorname{ran}(I-P(x))$ is invertible from $\operatorname{ran}(I-P(x))$ into $\operatorname{ran}\left(I-P\left(\phi^{n} x\right)\right)$; but $\operatorname{ran}(I-P(x))$ need not be finitedimensional. Such a concept appears in [24] and could produce numbers $\theta \in(-\infty, \kappa(\pi)] \cap \widetilde{\operatorname{Res}}(\pi)$ for the corresponding resolvent set $\widetilde{\operatorname{Res}}(\pi)$

\section{LYAPUNOV EXPONENTS AND MULTIPLICATIVE ERGODIC THEOREMS}

\subsection{Lyapunov Exponents}

Let $\pi=(\phi, \Phi)$ be a continuous skew-product flow over the compact metric space $X$. If the dynamical index of compactness $\kappa_{+}(\pi)$ is smaller than the maximal expansion $\bar{\mu}(\pi)$, we obtain a nontrivial exponential splitting $\mathcal{S}(\pi)=\left\{\mathcal{H}_{j}=\mathcal{H}_{\lambda_{j}, \mu_{j}}(\pi)\right\}$ as discussed in Theorem 2. We define Lyapunov exponents for $x \in X$ and $v \in E \backslash\{0\}$ as follows:

$$
\begin{aligned}
& \bar{\lambda}_{+}(x, v):=\limsup _{n \rightarrow+\infty} \frac{1}{n} \log \|\Phi(n, x) v\|, \\
& \underline{\lambda}_{+}(x, v):=\liminf _{n \rightarrow+\infty} \frac{1}{n} \log \|\Phi(n, x) v\| .
\end{aligned}
$$


We can also define the following Lyapunov exponents for $x \in X$ and $v \in \mathcal{B}^{-}(x) \backslash\{0\}$ with negative continuation $\left(x_{-1}, v_{-1}\right),\left(x_{-2}, v_{-2}\right), \ldots$ :

$$
\begin{aligned}
& \bar{\lambda}_{-}(x, v):=\limsup _{n \rightarrow-\infty} \frac{1}{n} \log \left\|v_{n}\right\|, \\
& \underline{\lambda}_{-}(x, v):=\liminf _{n \rightarrow-\infty} \frac{1}{n} \log \left\|v_{n}\right\| .
\end{aligned}
$$

If there is no such sequence $\left(x_{-n}, v_{-n}\right)$ then we set $\bar{\lambda}_{-}(x, v):=-\infty$.

It is then clear from construction of the exponential splitting that all the above Lyapunov exponents lie in the dynamical spectrum. More precisely (a proof can be found in [10, Theorem 6.48, Corollary 6.49, 25, 26]):

Proposition 2. Let $j_{0}$ be the minimal index $j$ such that $v$ has a nontrivial component in $\mathcal{H}_{j}(x)$ and $k_{0}$ the maximal $k$ such that $v$ has a nontrivial component in $\mathcal{H}_{k}(x)$ (assuming that both indices do exist). Then

$$
\begin{aligned}
& \lambda_{j_{0}} \leqslant \underline{\lambda}_{+}(x, v) \leqslant \bar{\lambda}_{+}(x, v) \leqslant \mu_{j_{0}}, \\
& \lambda_{k_{0}} \leqslant \underline{\lambda}_{-}(x, v) \leqslant \bar{\lambda}_{-}(x, v) \leqslant \mu_{k_{0}} .
\end{aligned}
$$

In particular, if $(x, v)$ belongs to finitely many $\mathcal{H}_{j}$ 's,

$$
\bar{\lambda}_{ \pm}(x, v), \underline{\lambda}_{ \pm}(x, v) \in\left[\lambda_{j}, \mu_{j}\right] \quad \text { if, and only if, } \quad v \in \mathcal{H}_{j}(x) \backslash\{0\} .
$$

\subsection{The Multiplicative Ergodic Theorems of Thieullen and Mañé}

Let now $v$ be a $\phi$-invariant Borel probability measure on $X$. We can consider for a continuous cocycle the pointwise analogue of the uniform quantities characterizing the cocycle (see Section 4.1):

$$
\begin{aligned}
\kappa(x) & :=\kappa(\pi)(x):=\liminf _{n \rightarrow+\infty} \frac{1}{n} \log \varrho(\Phi(n, x) \mid E), \\
\bar{\mu}(x) & :=\bar{\mu}(\pi)(x):=\limsup _{n \rightarrow+\infty} \frac{1}{n} \log \|\Phi(n, x) \mid E\| .
\end{aligned}
$$

The Subadditive Ergodic Theorem [27, Chapter 2] yields

$$
\begin{aligned}
\kappa_{v}(x) & :=\kappa(x)=\lim _{n \rightarrow+\infty} \frac{1}{n} \log \varrho(\Phi(n, x) \mid E), \\
\bar{\mu}_{\nu}(x) & :=\bar{\mu}(x)=\lim _{n \rightarrow+\infty} \frac{1}{n} \log \|\Phi(n, x) \mid E\|
\end{aligned}
$$

for $v$-a.e. $x \in X$. The number $\kappa_{v}(x)$ will be called the measurable index of compactness. We define $\mathcal{N}(x):=\bigcap_{n=1}^{\infty} \Phi\left(n, \phi^{-n} x\right) E$ for $x \in X$. If $\Phi(x)$ is injective for all $x \in X$, then every $v \in \mathcal{N}(x)$ has a unique inverse image 
under $\Phi\left(n, \phi^{-n} x\right)$ and we can consider orbits going to $-\infty$ in time. If $\bar{\mu}_{v}(x)>\kappa_{v}(x)$ on a set of positive measure, we obtain Multiplicative Ergodic Theorems on this set of positive measure. They were first proved by Mañé [4] for compact cocycles (see also [28, p. 263 ff.]); the general case is due to Thieullen [5]. We formulate the result here in a special case.

Theorem 3. (Multiplicative Ergodic Theorem). Let $X$ be a compact metric space, $\phi: X \rightarrow X$ a homeomorphism, v a $\phi$-invariant Borel probability measure on $X$ and $E$ a (real) Banach space. Let $\Phi$ be the cocycle over $\phi$ generated by the continuous mapping $\Phi: X \rightarrow \mathcal{L}(E)$. We moreover suppose that $\Phi(x)$ is injective for all $x \in X$. Then there exist a $\phi$-invariant Borel subset $Y_{v}$ (i.e. $\phi\left(Y_{v}\right)=Y_{v}$ ) of full measure (i.e. $v\left(Y_{v}\right)=v\left(\left\{x \mid \kappa_{v}(x)<\bar{\mu}_{v}(x)\right\}\right)$ ), $p_{v} \in \mathbb{N} \cup\{+\infty\}$, measurable functions $\lambda_{v}^{1}(x), \lambda_{v}^{2}(x), \ldots, \lambda_{v}^{p_{v}}(x)$, families of measurable subbundles $\mathcal{V}_{v}^{j}, \widehat{\mathcal{V}}_{v}^{j}$ such that for all $x \in Y_{v}$ and $j=1, \ldots, p_{v}$ (if $p_{v}=+\infty$ we do not claim the existence, e.g., of a number $\lambda_{v}^{p_{v}}$; we simply mean that there are infinitely many numbers $\left.\lambda_{v}^{j}(x)\right)$ :

(1) $\bar{\mu}_{v}(x)=\lambda_{v}^{1}(x)>\lambda_{v}^{2}(x)>\cdots>\lambda_{v}^{p_{v}}(x)>\kappa_{v}(x)$ (if $p_{v}=+\infty$, $\left.\lambda_{\nu}^{j}(x) \searrow \kappa_{v}(x)\right)$

(2) $\quad \lambda_{v}^{j}(\phi x)=\lambda_{v}^{j}(x), \quad \mathcal{V}_{v}^{j}(\phi x)=\mathcal{V}_{v}^{j}(x), \quad \widehat{\mathcal{V}}_{\nu}^{j}(\phi x)=\widehat{\mathcal{V}}_{v}^{j}(x)$; $\Phi(x) \mathcal{V}_{v}^{j}(x)=\mathcal{V}_{v}^{j}(\phi x), \quad \Phi(x) \widehat{\mathcal{V}}_{v}^{j}(x)=\widehat{\mathcal{V}}_{v}^{j}(\phi x)$;

(3) $\operatorname{dim}\left(\mathcal{V}_{\nu}^{j}(x)\right)<\infty$;

(4) $\widehat{\mathcal{V}}_{v}^{1}(x)=E, \quad \widehat{\mathcal{V}}_{v}^{j}(x)=\mathcal{V}_{v}^{j}(x) \oplus \widehat{\mathcal{V}}_{v}^{j+1}(x)$;

(5) if $v \in \mathcal{V}_{v}^{j}(x) \backslash\{0\}$ then $v \in \mathcal{N}(x)$ and $\lim _{n \rightarrow \pm \infty} \frac{1}{n} \log \|\Phi(n, x) v\|=$ $\lambda_{v}^{j}(x)$

(6) $\lim _{n \rightarrow+\infty} \frac{1}{n} \log \left\|\Phi(n, x) \mid \widehat{\mathcal{V}}_{\nu}^{j+1}(x)\right\|=\lambda_{v}^{j+1}(x) \quad$ (if $p_{v}$ is finite, we define $\left.\lambda_{v}^{p_{v}+1}:=\kappa_{v}\right)$;

(7) $\quad \limsup \frac{1}{n} \log \|\Phi(-n, x) v\|>-\lambda_{v}^{j}(x) \quad$ if $v \in \mathcal{N}(x) \cap \widehat{\mathcal{V}}_{v}^{j+1}(x)$.

Remark that, if we suppose $v$ ergodic, the Lyapunov exponents and the measurable index of compactness are $\phi$-invariant functions and thus constant. Thieullen's Theorem was generalized by Schaumlöffel [29], Schaumlöffel and Flandol [30].

If the generator $\Phi(x)$ of the cocycle is not injective, we can not expect an exponential splitting, even in finite dimensions. However, we can prove an analogous theorem with a flag decomposition of the state-space $E$ (see [5, Theorem 2.3]). More precisely Thieullen showed:

Theorem 4. (Multiplicative Ergodic Theorem). Let $X$ be a compact metric space, $\phi: X \rightarrow X$ a homeomorphism, $v$ a $\phi$-invariant Borel 
probability measure on $X$ and $E$ a (real) Banach space. Let $\Phi$ be the cocycle over $\phi$ generated by the continuous mapping $\Phi: X \rightarrow \mathcal{L}(E)$. Then there exist a $\phi$-invariant Borel subset $Y_{v}$ of full measure (i.e. $\phi\left(Y_{v}\right)=Y_{v}$ and $\left.v\left(Y_{v}\right)=v\left(\left\{x \mid \kappa_{v}(x)<\bar{\mu}_{v}(x)\right\}\right)\right), \quad p_{v} \in \mathbb{N} \cup\{+\infty\}$, measurable functions $\lambda_{v}^{1}(x), \lambda_{v}^{2}(x), \ldots, \lambda_{v}^{p_{v}}(x)$, families of measurable subbundles $\mathcal{V}_{v}^{j}$ such that for all $x \in Y_{v}$ and $j=1, \ldots, p_{v}$ :

(1) $\bar{\mu}_{\nu}(x)=\lambda_{v}^{1}(x)>\lambda_{v}^{2}(x)>\cdots>\lambda_{\nu}^{p_{v}}(x)>\kappa_{v}(x)$ (if $p_{v}=+\infty$, $\left.\lambda_{v}^{j}(x) \searrow \kappa_{v}(x)\right)$

(2) $\quad \lambda_{\nu}^{j}(\phi x)=\lambda_{v}^{j}(x), \quad \mathcal{V}_{v}^{j}(\phi x)=\mathcal{V}_{v}^{j}(x), \quad \Phi(x) \mathcal{V}_{v}^{j}(x)=\mathcal{V}_{v}^{j}(\phi x)$;

(3) $E=\mathcal{V}_{v}^{1}(x) \supset \mathcal{V}_{v}^{2}(x) \supset \cdots \supset \mathcal{V}_{v}^{p_{v}+1}(x)$ and $\operatorname{codim}\left(\mathcal{V}_{v}^{j}(x)\right)<\operatorname{codim}\left(\mathcal{V}_{v}^{j+1}(x)\right)<\infty$;

(4) $\lim _{n \rightarrow+\infty} \frac{1}{n} \log \|\Phi(n, x) v\|=\lambda_{v}^{j}(x) \quad$ if $v \in \mathcal{V}_{v}^{j}(x) \backslash \mathcal{V}_{\nu}^{j+1}(x)$;

(5) $\lim _{n \rightarrow+\infty} \frac{1}{n} \log \left\|\Phi(n, x) \mid \mathcal{V}_{v}^{j}(x)\right\|=\lambda_{v}^{j}(x)$ and, if $p_{v}$ is finite, $\lim _{n \rightarrow+\infty} \frac{1}{n} \log \left\|\Phi(n, x) \mid \mathcal{V}_{v}^{p_{v}+1}(x)\right\|=\kappa_{v}(x)$.

Remark 5. Proof of Theorem 4 is based on the construction of an enlarged state-space $\widetilde{E}$ by choosing

$$
\widetilde{E}:=\left\{\widetilde{v}=\left(v_{k}\right)_{k \geqslant 1} \in E^{\mathbb{N}} \mid \sup _{k \geqslant 1}\left\|v_{k}\right\|<+\infty\right\},
$$

equipped with the supremum norm. Pick a sequence $\left(\alpha_{k}\right)_{k \geqslant 1}$ of positive reals and define a skew-product flow $\tilde{\pi}$ over $\phi$ by

$$
\widetilde{\Phi}(x) \widetilde{v}:=\left(\Phi(x) v_{1}, \alpha_{1} v_{1}, \alpha_{2} v_{2}, \ldots\right), \quad(x, \widetilde{v}) \in X \times \widetilde{E} .
$$

Each operator $\widetilde{\Phi}(x)$ is injective. The sequence $\left(\alpha_{k}\right)$ can be chosen in such a way that the Lyapunov exponents of $\pi$ and $\tilde{\pi}$ agree [5, Lemma 4.2]. More precisely, $\left(\alpha_{k}\right)$ can be chosen such that

$$
\begin{aligned}
& \liminf _{n \rightarrow \infty} \frac{1}{n} \log \varrho(\widetilde{\Phi}(n, x)) \leqslant \liminf _{n \rightarrow \infty} \frac{1}{n} \log \varrho(\Phi(n, x)) \\
& \quad \leqslant \limsup _{n \rightarrow \infty} \frac{1}{n} \log \varrho(\Phi(n, x)) \leqslant \limsup _{n \rightarrow \infty} \frac{1}{n} \log \varrho(\widetilde{\Phi}(n, x)), \\
& \liminf _{n \rightarrow \infty} \frac{1}{n} \log \|\widetilde{\Phi}(n, x)\| \leqslant \liminf _{n \rightarrow \infty} \frac{1}{n} \log \|\Phi(n, x)\| \\
& \quad \leqslant \limsup _{n \rightarrow \infty} \frac{1}{n} \log \|\Phi(n, x)\| \leqslant \limsup _{n \rightarrow \infty} \frac{1}{n} \log \|\widetilde{\Phi}(n, x)\|, \\
& \liminf _{n \rightarrow \infty} \frac{1}{n} \log \|\widetilde{\Phi}(n, x) \widetilde{v}\| \leqslant \liminf _{n \rightarrow \infty} \frac{1}{n} \log \left\|\Phi(n, x) v_{1}\right\|
\end{aligned}
$$




$$
\leqslant \limsup _{n \rightarrow \infty} \frac{1}{n} \log \left\|\Phi(n, x) v_{1}\right\| \leqslant \limsup _{n \rightarrow \infty} \frac{1}{n} \log \|\widetilde{\Phi}(n, x) \widetilde{v}\| .
$$

We can apply Theorem 3 to the system $(X, v, \tilde{\pi}, \widetilde{E})$ and obtain a set $Y_{v}$ of full measure in $\left\{x \mid \kappa_{\nu}(x)<\bar{\mu}_{\nu}(x)\right\}$ with a corresponding flag decomposition $\widetilde{E}=\widehat{\mathcal{V}}_{v}^{1}(x) \supset \widehat{\mathcal{V}}_{v}^{2}(x) \supset \cdots$ for all $x \in Y_{v}$. The theorem follows projecting the spaces on $E$.

\subsection{Lyapunov and Dynamical Spectra}

Let now $\pi=(\phi, \Phi)$ be a continuous skew-product flow with nontrivial exponential splitting $\mathcal{S}(\pi)=\left\{\mathcal{H}_{j}:=\mathcal{H}_{\lambda_{j}, \mu_{j}}(\pi) \mid 1 \leqslant j \leqslant p\right\}$. According to Theorem 2 (Bounded Sets and Exponential Splittings) there are $\pi$-invariant, continuous subbundles $\widehat{\mathcal{H}}_{j}$ of $X \times E$ such that

$$
\widehat{\mathcal{H}}_{1}=X \times E, \quad \widehat{\mathcal{H}}_{j}=\mathcal{H}_{j} \oplus \widehat{\mathcal{H}}_{j+1} \quad \text { for } j=1, \ldots, p .
$$

Let $v$ be a $\phi$-invariant, ergodic Borel probability measure on $X$; if $\Phi(x)$ is injective for all $x$, Theorem 3 (Multiplicative Ergodic Theorem) gives us $v$-continuous subbundles $\mathcal{V}_{v}^{j}, \widehat{\mathcal{V}}_{\nu}^{j}$, a Borel subset $Y_{v}$ of full measure in $X$, an integer $p_{v}$ such that

$$
\widehat{\mathcal{V}}_{v}^{1}=Y_{v} \times E, \quad \widehat{\mathcal{V}}_{v}^{j}=\mathcal{V}_{v}^{j} \oplus \widehat{\mathcal{V}}_{v}^{j+1} \quad \text { for } j=1, \ldots, p_{v}
$$

and corresponding Lyapunov exponents $\lambda_{\nu}^{j}$. Let $\theta_{j}$ be in $\operatorname{Res}(\pi), \theta_{j}<\lambda_{j}$ and $k_{j}=k_{j}(v)$ be the integer such that

$$
\begin{aligned}
& \max \left\{\kappa_{v}, \lambda_{v}^{k_{j}+1}\right\} \leqslant \mu_{j+1}<\theta_{j}<\lambda_{j} \leqslant \lambda_{v}^{k_{j}}, \quad \text { if } j<p, \\
& \max \left\{\kappa_{v}, \lambda_{v}^{k_{p}+1}\right\} \leqslant \kappa_{+}(\pi)<\theta_{p}<\lambda_{p} \leqslant \lambda_{v}^{k_{p}}, \quad \text { if } p \text { is finite. }
\end{aligned}
$$

The measurable subbundles are then the traces on $Y_{v}$ of the continuous subbundles (see [10, Proposition 8.10]).

Proposition 3. For all $1 \leqslant j \leqslant p$ we have:

(1) $\widehat{\mathcal{V}}_{\nu}^{k_{j}+1}(x)=\widehat{\mathcal{H}}_{j+1}(x)$ for all $x \in Y_{\nu}$;

(2) $\bigoplus \mathcal{V}_{v}^{\ell}(x)=\mathcal{H}_{j}(x) \quad$ for all $x \in Y_{\nu}$.

$\ell: \lambda_{v}^{\ell} \in\left[\lambda_{j}, \mu_{j}\right]$

In particular, the sum of the dimensions of the $\mathcal{V}_{v}^{\ell}(x)$ does not depend on the measure $v$.

(3) If, moreover, $p$ is finite, then

$$
\bigoplus_{\ell: \lambda_{v}^{\ell} \in\left(\kappa_{\nu}, \kappa_{+}(\pi)\right]} \mathcal{V}_{\nu}^{\ell}(x) \oplus \widehat{\mathcal{V}}_{\nu}^{p_{v}+1}(x)=\widehat{\mathcal{H}}_{p+1}(x) \quad \text { for all } x \in Y_{\nu} .
$$

(Last formula can also be interpreted for $p=+\infty$.) 
Corollary 2. Let $\pi$ be a continuous skew-product flow with infinitely many compact intervals $\left[\lambda_{j}, \mu_{j}\right]$ in the dynamical spectrum $\Sigma(\pi)$. Then $\mu_{j} \searrow \kappa(\pi)=\kappa_{+}(\pi)$.

Proof of Corollary 2. By Theorem 3, the Lyapunov exponents $\lambda_{v}^{j}$ converge to $\kappa_{\nu} \leqslant \kappa(\pi) \nu$-a.s. for any invariant, ergodic Borel probability measure $v$. Therefore, in view of Proposition 3, it is impossible that the $\mu_{j}$ 's converge to a number larger than $\kappa(\pi)$ : We would get Lyapunov exponents accumulating at a number larger than $\kappa_{\nu}$.

\section{Remark 6.}

(i) We obtain a similar result if we do not suppose $v$ ergodic.

(ii) We also get a similar result in the framework of Theorem 4 and Corollary 2 remains valid: the subbundles $\mathcal{V}_{v}^{j}$ giving the flag decomposition are such that

$$
\widehat{\mathcal{H}}_{j}(x)=\mathcal{V}_{\nu}^{k_{j}}(x)=\mathcal{H}_{j}(x) \oplus \mathcal{V}_{\nu}^{k_{j}+1}(x) \text { for all } x \in Y_{\nu} .
$$

(iii) If the Banach space $E$ is finite-dimensional and the skew-product flow $\pi$ invertible, then we have

$$
\mu_{j}=\sup \lambda_{v}^{\ell}, \quad \lambda_{j}=\inf \lambda_{v}^{\ell},
$$

where $\ell$ runs over all integers $k$ with $\lambda_{v}^{k} \in\left[\lambda_{j}, \mu_{j}\right]$ and $v$ over the $\phi$-invariant, ergodic Borel probability measures on $X$; the extrema are moreover attained. (A similar result holds for separable Hilbert spaces and continuous cocycles with values in the algebra of compact operators, see [8, 9 or 10 , Theorem 8.15$]$.) Applying this result to the restriction of the skew-product flow to the subbundles $\mathcal{H}_{j}$ we find a similar result in the context of Theorems 3 and 4. It is moreover proved in [10, Theorem 8.15] that $\bar{\mu}(\pi)=\sup \lambda_{v}^{\ell}$ in the Hilbert-space framework cited above. We generalize this result below to the Banach space setting and prove moreover that the supremum is attained.

Theorem 5. Let $\phi$ be a homeomorphism of the compact metric space $X$ and $\Phi: X \rightarrow \mathcal{L}(E)$ a continuous map generating a skew-product flow $\pi$ over $X$. We moreover suppose that $\Phi(x)$ is injective for all $x \in X$. We have then

$$
\kappa(\pi)=\sup \kappa_{v},
$$


where the supremum is taken over the $\phi$-invariant, ergodic Borel probability measures $v$ on $X$. Moreover, the number $\kappa_{+}(\pi)$ appearing in Theorem 2 satisfies

$$
\kappa_{+}(\pi)=\sup \lambda_{v}^{\ell}
$$

where the supremum is taken over the $\phi$-invariant, ergodic Borel probability measures $v$ on $X$ and the Lyapunov exponents $\lambda_{v}^{\ell}$ of the system $(\pi, v)$ such that $\lambda_{v}^{\ell} \leqslant \kappa_{+}(\pi)$. The suprema (5) and (6) are moreover attained.

We note that similar results are obtained if we do not suppose that all maps $\Phi(x)$ are injective (see Theorem 4 and Remark 5).

Corollary 3. We have in particular:

$$
\bar{\mu}(\pi)=\sup \bar{\mu}_{v}
$$

where the supremum is taken over the $\phi$-invariant, ergodic Borel probability measures $v$ on $X$, and $\bar{\mu}_{v}$ is the largest Lyapunov exponent of $(\pi, v)$ (or the measurable index of compactness $\kappa_{v}$ if there are no Lyapunov exponents). The supremum is moreover attained.

Proof of Theorem 5. We follow the general strategy of [8, 9]. We first prove that $\kappa_{+}(\pi)=\sup \lambda_{v}^{\ell}$. Restricting the dynamics to invariant subspaces if necessary, we can assume without loss of generality that $\kappa_{+}(\pi)=\bar{\mu}(\pi)>$ $-\infty$. First note that, by Theorem 2 ,

$$
\bar{\mu}(\pi)=\lim _{n \rightarrow \infty} \frac{1}{n} \log \sup _{x \in X}\|\Phi(n, x)\| .
$$

Moreover, the largest Lyapunov exponent $\bar{\mu}_{v}$ with respect to an ergodic measure $v$ is given for a point $x$ in a set $Y_{v}$ of full measure in $X$ by

$$
\bar{\mu}_{\nu}=\lim _{n \rightarrow \infty} \frac{1}{n} \log \|\Phi(n, x)\| \leqslant \bar{\mu}(\pi) .
$$

We now construct an ergodic probability measure $v$ such that $\bar{\mu}_{v}=\bar{\mu}(\pi)$. The strategy is to construct a measure $\eta$ on the compactification of a "projective bundle" $X \times \mathbf{P}(E)$ to be defined, and to obtain $v$ as the projection of $\eta$ on $X$.

We first projectivize the vector space $E$, identifying lines through the origin in $E$. We get a space $\mathbf{P}(E)$, and equip this space with the strongest topology making the projection from $E$ to $\mathbf{P}(E)$ continuous. We then put on $\mathcal{X}:=X \times \mathbf{P}(E)$ the product topology. In this way, $\mathcal{X}$ becomes a metrizable topological space. 
The first idea is to "scalarize" the cocycle $\Phi$ : Since $\Phi(x)$ is injective for $x \in X$, we can define a scalar-valued function $f$ on $\mathcal{X}$ by

$$
f(x, \mathbf{v}):=\log \|\Phi(x) v\|, \quad \text { where } v \in \mathbf{v} \text { with }\|v\|=1 .
$$

Note that $f \in \mathcal{C}(\mathcal{X}, \mathbb{R})$.

The next step is to "projectivize" the homeomorphism $\phi$ of $X$; we define a map $\psi$ on $\mathcal{X}$ as follows:

$$
\psi(x, \mathbf{v}):=(\phi x, \Phi(x) \mathbf{v})
$$

Each map $\Phi(x)$ is linear, so maps lines to lines in $E$ and is thus defined - and continuous - on $\mathbf{P}(E)$; so $\psi \in \mathcal{C}(\mathcal{X})$. Let us observe that

$$
\psi^{n}(x, \mathbf{v})=\left(\phi^{n} x, \Phi(n, x) \mathbf{v}\right) \text { for } n \geqslant 1 .
$$

We now choose points $x_{n} \in X, v_{n} \in \mathbf{v}_{n} \in \mathbf{P}(E)$ with $\left\|v_{n}\right\|=1, n \geqslant 1$, such that

$$
\left\|\Phi\left(n, x_{n}\right) v_{n}\right\| \geqslant \frac{1}{2} \sup _{x \in X}\|\Phi(n, x)\| .
$$

Let $\beta \mathbf{P}(E)$ denote the Stone-Čech compactification of $\mathbf{P}(E)$ (see [6, $\S \mathrm{V} .6])$. We denote by $\mathcal{C}_{b}(\mathcal{X}, \mathbb{R})$ the space of bounded continuous functions on $\mathcal{X}$ and define $\beta \mathcal{X}:=X \times \beta \mathbf{P}(E)$. Since $\mathcal{C}_{b}(\mathcal{X}, \mathbb{R})^{*}=M(\beta \mathcal{X})$, the space of regular Borel measures on $\beta \mathcal{X}$, we can define a probability measure $\eta_{n}$ on $\beta \mathcal{X}$ by

$$
\eta_{n}(g):=\frac{1}{n} \sum_{j=0}^{n-1} g\left(\psi^{j}\left(x_{n}, \mathbf{v}_{n}\right)\right), \quad g \in \mathcal{C}_{b}(\mathcal{X}, \mathbb{R}) .
$$

By passing to a subsequence if necessary we can assume that $\left(\eta_{n}\right)$ converges in the weak-star topology to some Borel measure $\eta$. So,

$$
\eta_{n}(g) \longrightarrow \eta(g) \text { for all } g \in \mathcal{C}_{b}(\mathcal{X}, \mathbb{R}) .
$$

It is important to note that $f$ is not necessarily bounded on $\mathcal{X}$.

Claim 1. The function $f$ represents a well-defined element $f^{\beta}$ in the space $L^{1}(\beta \mathcal{X}, \eta)$ and

$$
\eta\left(f^{\beta}\right) \geqslant \bar{\mu}(\pi) .
$$


Proof of the Claim. We first remark that $\mathcal{X}$ has full measure in $\beta \mathcal{X}$. Therefore, $f$ can be viewed as an $\eta_{n}$ - or $\eta$-measurable function $f^{\beta}$ on $\beta \mathcal{X}$. However, $f^{\beta}$ is $\eta_{n}$-integrable for all $n \geqslant 1$ and

$$
\eta_{n}\left(f^{\beta}\right)=\frac{1}{n} \sum_{j=0}^{n-1} \log \frac{\left\|\Phi\left(j+1, x_{n}\right) v_{n}\right\|}{\left\|\Phi\left(j, x_{n}\right) v_{n}\right\|} .
$$

We thus obtain

$$
\eta_{n}\left(f^{\beta}\right)=\frac{1}{n} \log \left\|\Phi\left(n, x_{n}\right) v_{n}\right\| \longrightarrow \bar{\mu}(\pi) .
$$

We define for each $N \geqslant 1$ a function $f_{N}$ on $\mathcal{X}$ by $f_{N}:=\max \{f,-N\}$; $f_{N} \in \mathcal{C}_{b}(\mathcal{X}, \mathbb{R})$ and has thus a unique continuous extension $f_{N}^{\beta}$ to $\beta \mathcal{X}$. If we let $M$ be $M:=\log \sup _{x}\|\Phi(x)\|<\infty$, then

$$
f^{\beta}(x, \mathbf{v}) \leqslant f_{N}^{\beta}(x, \mathbf{v}) \leqslant M \quad \text { for }(x, \mathbf{v}) \in \mathcal{X} \text { and } N \geqslant 1 .
$$

However, since $f_{N}^{\beta} \in \mathcal{C}(\beta \mathcal{X}, \mathbb{R})$,

$$
\eta\left(f_{N}^{\beta}\right)=\lim _{n \rightarrow \infty} \eta_{n}\left(f_{N}^{\beta}\right) \geqslant \lim _{n \rightarrow \infty} \eta_{n}\left(f^{\beta}\right)=\bar{\mu}(\pi) .
$$

On the other hand, $f_{N}^{\beta} \searrow f^{\beta}$ pointwise on $\mathcal{X}$, i.e., $\eta$-almost everywhere on $\beta \mathcal{X}$. So, by the Monotone Convergence Theorem, $f^{\beta} \in L^{1}(\beta \mathcal{X}, \eta)$ and

$$
\eta\left(f^{\beta}\right)=\lim _{N \rightarrow \infty} \eta\left(f_{N}^{\beta}\right) \geqslant \bar{\mu}(\pi) .
$$

Let $\psi^{\beta}$ be the unique continuous extension of $\psi$ to $\beta \mathcal{X}$. Remark that $\eta$ is $\psi^{\beta}$-invariant since $\eta(g \circ \psi)=\eta(g)$ for all $g \in \mathcal{C}_{b}(\mathcal{X}, \mathbb{R})$. The Birkhoff Ergodic Theorem gives a subset $\beta \mathcal{X}_{\eta}$ of full measure in $\beta \mathcal{X}$ such that, for each point $\xi$ in $\beta \mathcal{X}_{\eta}$,

$$
\overline{f^{\beta}}(\xi):=\lim _{n \rightarrow \infty} \frac{1}{n} \sum_{j=0}^{n-1} f^{\beta}\left(\left(\psi^{\beta}\right)^{j}(\xi)\right) \quad \text { exists and } \eta\left(\overline{f^{\beta}}\right)=\eta\left(f^{\beta}\right) .
$$

Let $p$ (respectively, $\widetilde{p}$ ) be the projection of $\mathcal{X}$ onto $X$ (respectively, $\mathbf{P}(E)$ ) and let $p^{\beta}: \beta \mathcal{X} \rightarrow X$ (respectively, $\widetilde{p}^{\beta}: \beta \mathcal{X} \rightarrow \beta \mathbf{P}(E)$ ) be the continuous extension of $p$ (respectively, $\widetilde{p}$ ) to $\beta \mathcal{X}$. We define measures $v$ and $\widetilde{v}$ on $X$ and each fiber $\beta \mathcal{X}(x)=\beta \mathbf{P}(E)$, respectively, by

$$
v:=p_{*}^{\beta} \eta, \quad \widetilde{v}:=\widetilde{p}_{*}^{\beta} \eta .
$$


Note that $v$ is $\phi$-invariant since $\eta$ is $\psi^{\beta}$-invariant and that $\mathcal{X}_{\eta}:=\beta \mathcal{X}_{\eta} \cap \mathcal{X}$ is a set of full $\eta$-measure in $\beta \mathcal{X}$. Decomposing $v$ into ergodic components if necessary, we can suppose without loss of generality that $v$ is ergodic. The Multiplicative Ergodic Theorem for $v$ and $\pi$ gives a subset $Y_{v}$ of full measure in $X$ such that

$$
\lim _{n \rightarrow \infty} \frac{1}{n} \log \|\Phi(n, x)\|=\bar{\mu}_{\nu} \quad \text { for all } x \in Y_{\nu} .
$$

So, if $(x, \mathbf{v}) \in \mathcal{Y}_{\eta}:=p\left(\mathcal{X}_{\eta}\right) \cap Y_{v} \times \widetilde{p}\left(\mathcal{X}_{\eta}\right)$ and $v \in \mathbf{v}$ with $\|v\|=1$, then

$$
\begin{aligned}
\bar{\mu}_{v} & \geqslant \lim _{n \rightarrow \infty} \frac{1}{n} \log \|\Phi(n, x) v\|=\lim _{n \rightarrow \infty} \frac{1}{n} \sum_{j=0}^{n-1} f^{\beta}\left(\psi^{j}(x, \mathbf{v})\right) \\
& =\overline{f^{\beta}}(x, \mathbf{v}) .
\end{aligned}
$$

The argument below is the main difference with [10, Theorem 8.15]. Denoting the product measure on $\beta \mathcal{X}$ by $v \otimes \widetilde{v}$, we remark that $\mathcal{Y}_{\eta}$ is a subset of full $(v \otimes \widetilde{v})$-measure in $\beta \mathcal{X}$. The measure $\eta$ is moreover absolutely continuous with respect to $v \otimes \widetilde{v}$ since

$$
\eta(\mathcal{Z})^{2} \leqslant(v \otimes \widetilde{v})(\mathcal{Z}) \text { for every Borel subset } \mathcal{Z} \text { of } \beta \mathcal{X} .
$$

Hence, by the Radon-Nikodým Theorem, there is a nonnegative function $a \in L^{1}(\beta \mathcal{X}, v \otimes \widetilde{v})$ such that

$$
\int_{\mathcal{Z}} g d \eta=\int_{\mathcal{Z}} g \cdot a d(v \otimes \widetilde{v})
$$

for every Borel subset $\mathcal{Z}$ of $\beta \mathcal{X}$, whenever the measurable function $g$ is such that $\int_{\mathcal{Z}} g d \eta$ exists. Moreover, $(\nu \otimes \widetilde{v})(a)=1$ and thus, since $\overline{f^{\beta}} \leqslant \bar{\mu}_{v}$ on a set of full $(v \otimes \widetilde{v})$-measure,

$$
\bar{\mu}(\pi) \leqslant \eta\left(f^{\beta}\right)=\eta\left(\overline{f^{\beta}}\right)=(\nu \otimes \widetilde{v})\left(\overline{f^{\beta}} \cdot a\right) \leqslant \bar{\mu}_{v} \cdot(\nu \otimes \widetilde{v})(a)=\bar{\mu}_{\nu} .
$$

The supremum in $\kappa_{+}(\pi)=\sup \lambda_{v}^{\ell}$ is therefore attained.

The proof of $\kappa(\pi)=\sup \kappa_{v}$ is similar and we only sketch it. Let $2^{E}$ be the set of all nonempty bounded subsets of $E$. We can put on $2^{E}$ a vector space structure by defining

$$
\begin{aligned}
& F+G:=\{u+v \mid u \in F, v \in G\}, \\
& t F:=\{t u \mid u \in F\}, \quad t \in \mathbb{R}, \quad F, G \in 2^{E} .
\end{aligned}
$$


Let $2_{c}^{E}$ be the subspace of relatively compact sets in $2^{E}$; the Kuratowski function $\alpha$ defines a norm on the quotient space $2^{E} / 2_{c}^{E}$ since $\alpha(F)=0$, $F \in 2^{E}$, if and only if $F$ is relatively compact in $E$. The next step is to projectivize the normed space $\left(2^{E} / 2_{c}^{E}, \alpha\right)$, identifying lines through the origin in $2^{E} / 2_{c}^{E}$. We get a space $\mathbf{P}\left(2^{E} / 2_{c}^{E}\right)$ and we consider the projective bundle $\mathcal{X}:=X \times \mathbf{P}\left(2^{E} / 2_{c}^{E}\right)$.

We claim that we can suppose without loss of generality that

$$
\alpha(\Phi(x) F)>0 \quad \text { whenever } \quad \alpha(F)>0, \quad x \in X, F \in 2^{E} .
$$

To prove this, consider if necessary the extension $\widetilde{\Phi}$ of $\Phi$ as in Remark 5 . We first note that

$$
\sup _{i \in \mathbb{N}} \alpha\left(F_{i}\right) \leqslant \alpha\left(\prod_{i \in \mathbb{N}} F_{i}\right), \quad \text { where } F_{i} \in 2^{E} \text { for all } i .
$$

To see this, we define for $\epsilon>0$ a number $r_{\epsilon}$ by $r_{\epsilon}:=\alpha\left(\prod_{i} F_{i}\right)+\epsilon$. There exist then a positive integer $k$ and vectors $\widetilde{u}^{(j)}=\left(u_{1}^{(j)}, u_{2}^{(j)}, \ldots\right) \in \prod_{i} F_{i}$, $1 \leqslant j \leqslant k$, such that

$$
\prod_{i \in \mathbb{N}} F_{i} \subset \bigcup_{j=1}^{k} B_{\widetilde{E}}\left(\widetilde{u}^{(j)}, r_{\epsilon}\right)=\bigcup_{j=1}^{k} \prod_{i \in \mathbb{N}} B_{E}\left(u_{i}^{(j)}, r_{\epsilon}\right) \subset \prod_{i \in \mathbb{N}} \bigcup_{j=1}^{k} B_{E}\left(u_{i}^{(j)}, r_{\epsilon}\right) .
$$

Each set $F_{i}$ can thus be covered by finitely many balls of radius not exceeding $r_{\epsilon}$ and with centers in $F_{i}$. Therefore,

$$
\sup _{i} \alpha\left(F_{i}\right) \leqslant r_{\epsilon}
$$

and (10) follows if we let $\epsilon$ tend to zero.

Let $F \in 2^{E}$ be such that $\alpha\left(F^{\mathbb{N}}\right)>0$; then, by Tychonoff's Theorem, $\alpha(F)>0$ and we thus get from (10) for $x \in X$ :

$$
\alpha\left(\widetilde{\Phi}(x) F^{\mathbb{N}}\right)=\alpha\left(\Phi(x) F \times \alpha_{1} F \times \alpha_{2} F \times \cdots\right) \geqslant \alpha(F) \sup _{i} \alpha_{i}>0,
$$

from which (9) follows. We can hence define a function $f$ on $\mathcal{X}$ by

$$
f(x, \mathbf{F}):=\log \alpha(\Phi(x) F), \quad \text { where } F \in \mathbf{F} \text { with } \alpha(F)=1 .
$$

Note that $f \in \mathcal{C}(\mathcal{X}, \mathbb{R})$ and $\Phi(x) \in \mathcal{L}\left(2^{E} / 2_{c}^{E}, \alpha\right)$ for $x \in X$.

We define as above a continuous mapping $\psi$ on $\mathcal{X}$ by

$$
\psi(x, \mathbf{F}):=(\phi x, \Phi(x) \mathbf{F})
$$


and choose points $x_{n} \in X, n \geqslant 1$, such that

$$
\alpha\left(\Phi\left(n, x_{n}\right) B\right) \geqslant \frac{1}{2} \sup _{x \in X} \varrho(\Phi(n, x)) .
$$

Here, $B$ denotes the unit ball in $E$. The proof can be completed as above, working with the Stone-Čech compactification of $\mathbf{P}\left(2^{E} / 2_{c}^{E}\right)$. We simply note the following fact used in step (8):

$$
\varrho(\Phi(x))=\sup _{\alpha(F)=1} \alpha(\Phi(x) F) .
$$

The upper bound follows from $\alpha(B)=1$ and the definition of $\varrho$; in order to prove the lower bound, it is sufficient to note that, given $\epsilon>0, F \in 2^{E}$ can be covered by a finite number $k$ of balls $B_{j}$ of radius $r_{\epsilon}:=\alpha(F)+\epsilon$ and centered in $u_{j} \in F, 1 \leqslant j \leqslant k$. Hence,

$$
\Phi(x) F \subset \bigcup_{j=1}^{k} \Phi(x) B_{j}=\bigcup_{j=1}^{k}\left(\Phi(x)\left\{u_{j}\right\}+\Phi(x)\left(r_{\epsilon} B\right)\right)
$$

and we get

$$
\alpha(\Phi(x) F) \leqslant \max _{1 \leqslant j \leqslant k}\left\{\alpha\left(\Phi(x)\left\{u_{j}\right\}\right)+r_{\epsilon} \alpha(\Phi(x) B)\right\}=r_{\epsilon} \varrho(\Phi(x)) .
$$

Therefore, if we let $\epsilon$ tend to zero and suppose $\alpha(F)>0$,

$$
\frac{\alpha(\Phi(x) F)}{\alpha(F)} \leqslant \varrho(\Phi(x))
$$

and the claim follows.

\section{ACKNOWLEDGMENTS}

Finally I would like to thank the referee for the careful reading of the manuscript, the useful suggestions, remarks and questions.

\section{REFERENCES}

1. Oseledec, V. I. (1968). A multiplicative ergodic theorem. Lyapunov characteristic numbers for dynamical systems. Trans. Moscow Math. Soc. 19, 197-221.

2. Ruelle, D. (1982). Characteristic exponents and invariant manifolds in Hilbert space. Ann. Math. 115, 243-290.

3. Nussbaum, R. D. (1970). The radius of the essential spectrum. Duke Math. J. 37, 473-478.

4. Mañé, R. (1981). Lyapunov exponents and stable manifolds for compact transformations. In Palis, J., Jr. (ed.), Geometric Dynamics, Proceedings, Rio de Janeiro 1981, Volume 1007 of Lecture Notes in Mathematics, Springer-Verlag, Berlin, pp. 522-577. 
5. Thieullen, P. (1987). Fibrés dynamiques asymptotiquement compacts, exposants de Lyapunov. Entropie. Dimension. Ann. Inst. H. Poincaré, Anal. non linéaire 4(1), 49-97.

6. Berger, A., and Siegmund, S. (2003). On the gap between random dynamical systems and continuous skew-products. J. Dyn. Diff. Eq. 15, 237-279.

7. Sacker, R. J., and Sell, G. R. (1994). Dichotomies for linear evolutionary equations in Banach spaces. J. Diff. Eq. 113(1), 17-67.

8. Latushkin, Y. D., and Stëpin, A. M. (1991). Weighted translation operators and linear extensions of dynamical systems. Russ. Math. Surv. 46(2), 95-165.

9. Johnson, R. A., Palmer, K. J., and Sell, G. R. (1987). Ergodic properties of linear dynamical systems. SIAM J. Math. Anal. 18(1), 1-33.

10. Chicone, C., and Latushkin, Y. (1999). Evolution Semigroups in Dynamical Systems and Differential Equations, Volume 70 of Mathematical Surveys and Monographs, American Mathematical Society, Providence, RI.

11. Kuratowski, C. (1930). Sur les espaces complets. Fund. Math. 15, 301-309.

12. Walters, P. (1982). An Introduction to Ergodic Theory, Volume 79 of Graduate Texts in Mathematics, Springer-Verlag, Berlin.

13. Sacker, R. J., and Sell, G. R. (1974). Existence of dichotomies and invariant splittings for linear differential systems I. J. Diff. Eq. 15(3), 429-458.

14. Sacker, R. J., and Sell, G. R. (1976). Existence of dichotomies and invariant splittings for linear differential systems II. J. Diff. Eq. 22(2), 478-496.

15. Sacker, R. J., and Sell, G. R. (1976). Existence of dichotomies and invariant splittings for linear differential systems III. J. Diff. Eq. 22(2), 497-522.

16. Mañé, R. (1977). Quasi-Anosov diffeomorphisms and hyperbolic manifolds. Trans. Am. Math. Soc. 229, 351-370.

17. Franks, J. M., and Robinson, C. (1976). A quasi-Anosov diffeomorphism that is not Anosov. Trans. Amer. Math. Soc. 223, 267-278.

18. Selgrade, J. F. (1975). Isolated invariant sets for flows on vector bundles. Trans. Am. Math. Soc. 203, 359-390.

19. Chow, S.-N., and Leiva, H. (1994). Dynamical spectrum for time dependent linear systems in Banach spaces. Jpn. J. Ind. Appl. Math. 11, 379-415.

20. Voutaz, É. (2001). Hyperbolicity of the Renormalization Operator for Critical $\mathcal{C}^{r}$ Cirlce Mappings, Diss. ETH No. 14363, ETH, Zürich.

21. Holschneider, M. (1996). Wavelet analysis of transfer operators acting on $n$-dimensional Hölder Besov Zygmund Triebel spaces. Preprint mp_arc 96-169.

22. Baladi, V., and Holschneider, M. (1999). Approximation of nonessential spectrum of transfer operators. Nonlinearity 12(3), 525-538.

23. Magalhães, L. T. (1987). The spectrum of invariant sets for dissipative semiflows. In Chow and Hale (eds.), Dynamics of Infinite Dimensional Systems, Volume 37 of NATO ASI Series F: Computer and Systems Sciences, Springer-Verlag, Berlin, pp. 161-168.

24. Latushkin, Y. D., Montgomery-Smith, S. J., and Randolph, T. W. (1996). Evolutionary semigroups and dichotomy of linear skew-product flows on locally compact spaces with Banach fibers. J. Diff. Eq. 125(1), 73-116.

25. Sacker, R. J., and Sell, G. R. (1978). A spectral theory for linear differential systems. J. Diff. Eq., 27(3), 320-358.

26. Chow, S.-N., and Leiva, H. (1995). Existence and roughness of the exponential dichotomy for skew-product semiflow in Banach spaces. J. Diff. Eq. 120(2) 429-477.

27. Pollicott, M. (1993). Lectures on Ergodic Theory and Pesin Theory on Compact Manifolds, Volume 180 of London Mathematical Society Lecture Notes, Cambridge University Press, Cambridge. 
28. Mañé, R. (1981). Ergodic Theory and Differentiable Dynamics, Volume 8 of Ergebnisse der Mathematik und ihrer Grenzgebiete, Springer-Verlag, Berlin.

29. Schaumlöffel, K. (1991). Multiplicative ergodic theorems in infinite dimensions. In Arnold, L., Crauel, H., and Eckmann, J. P. (eds.), Lyapunov Exponents, Proceedings, Oberwolfach 1990, Volume 1486 of Lecture Note in Mathematics, Springer-Verlag, Berlin, pp. 187-195.

30. Schaumlöffel, K., and Flandoli, F. (1991). A multiplicative ergodic theorem with applications to a first order stochastic hyperbolic equation in a bounded domain. Stoch. Stoch. Rep. 34(2), 241-255. 